\title{
Adjustment of Some Atmospheric Statistics to Satisfy Physical Conditions
}

\author{
C. Eugene Buel. \\ Kaman Sciences Corp., Colorado Springs, Colo. 80907
}

(Manuscript received 23 March 1972, in revised form 14 August 1972)

ABSTRACT

\begin{abstract}
A relation between the coefficients of variation for pressure, density and temperature is derived which must be satisfied if these statistical parameters are those of an atmosphere that satisfies the perfect gas law and the hydrostatic equation. Data on these parameters for Cape Kennedy, Fla., are adjusted on the basis of this relation. Corresponding adjusted values of the correlation coefficients $r_{p} r, r_{T_{\rho}}, r_{\rho p}$ are obtained. A parameter that measures the depth of pressure perturbation systems is also obtained for the levels from the surface to $90 \mathrm{~km}$.
\end{abstract}

\section{Introduction}

It was pointed out in Buell (1954) that the equation of state and the hydrostatic equation implied several specific relations connecting statistical parameters which describe properties of the atmosphere. Some of these relations that depend on the equation of state were later reviewed (Buell, 1970) in more detail. Minzner and Morgenstern (1971) checked the extent to which some of these relations were satisfied in the upper stratosphere. The relations between statistical parameters derived from the equation of state and hydrostatic equation are as valid as the latter relations themselves (if no additional assumptions are made in developing these relations). Since the statistical parameters involved are subject to so many sources of error, it would be a better procedure to use these relations to adjust the statistical parameters so that the adjusted values satisfy the required relationships.

To illustrate our point, some relations are derived from the equation of state and hydrostatic equation which connect the coefficients of variation for pressure, $\sigma_{p} / \bar{p}$, density, $\sigma_{\rho} / \bar{\rho}$, and temperature, $\sigma_{T} / \bar{T}$. The coefficients of variation were used, rather than the standard deviations since values of $\sigma_{p} / \bar{p}$ and $\sigma_{\rho} / \bar{\rho}$ do not vary over so wide a range as $\sigma_{p}$ and $\sigma_{\rho}$ alone.

We first derive the required relations, outline the adjustment procedure, and then apply the method to published data on the coefficients of variation for Cape Kennedy, Fla. The correlation coefficients $r_{p t^{\prime}}, r_{T^{\prime} \rho}, r_{\rho p}$ are computed from the adjusted values. The depth of pressure perturbation systems in the atmosphere is computed from the adjusted coefficients of variation and correlation coefficients as a function of altitude.

\section{Use of the hydrostatic equation}

Let $\xi=p^{\prime} / \bar{p}, \eta=\rho^{\prime} / \bar{\rho}, \zeta=T^{\prime} / \bar{T}$, where $\bar{p}, \bar{\rho}, \bar{T}$ are mean values and $p^{\prime}, \rho^{\prime}, T^{\prime}$ are departures from the re- spective means. Then the gas equation becomes [see Buell (1970), for the effect of the approximations]:

$$
\xi=\eta+\zeta .
$$

The hydrostatic equation, in terms of departure from the mean, may be written

$$
\partial(\bar{p} \xi) / \partial z=-g(\eta \bar{\rho}) .
$$

Since the hydrostatic equation holds for the means as well as for the departure from the means, it follows that

$$
H_{p}(\partial \xi / \partial z)=\zeta,
$$

where $H_{p}=R \bar{T} / g$ is the pressure scale height (about $8 \mathrm{~km}$ ). Multiplying (2) by $\xi$ and averaging leads to

or

$$
H_{p}\left(\partial \bar{\xi}^{2} / \partial z\right)=2(\overline{\xi \zeta})
$$

$$
H_{p}\left\{\partial\left(\sigma_{p} / \bar{p}\right) / \partial z\right\}=\left(\sigma_{T} / \bar{T}\right) r_{p T},
$$

where $r_{p T}$ is the correlation coefficient relating pressure and temperature at a point. From Buell (1954, 1970), $2(\overline{\xi \zeta})=\overline{\xi^{2}}+\overline{\zeta^{2}}-\overline{\eta^{2}}$, and making the substitutions, $U=\overline{\xi^{2}}$ $=\left(\sigma_{p} / \bar{p}\right)^{2}, V=\overline{\eta^{2}}=\left(\sigma_{p} / \bar{\rho}\right)^{2}, W=\overline{\zeta^{2}}=\left(\sigma_{T} / \bar{T}\right)^{2}$, one obtains from (2a)

$$
H_{p}(\partial U / \partial z)=U+W-V .
$$

This differential equation must be satisfied if the quantities $\sigma_{p}, \sigma_{\rho}, \sigma_{T}, \bar{p}, \bar{\rho}, \bar{T}$ and the implied quantities $U, V, W$ are to represent conditions in a physically realizable atmosphere in which the gas equation and hydrostatic equation are valid.

\section{Equations for adjustment}

In order to adjust the observed statistical quantities to satisfy (3) a certain amount of arbitrariness is involved. First, it is required that the adjustments be the smallest necessary to bring about the desired 
result. If $u, v, v$ are adjustments and $U^{0}, V^{0}, W^{0}$ are the observed parameters so that $U=U^{0}+u$, etc., then the weighted sum of squares of the adjustments to be minimized is

$$
F=\sum_{i=1}^{n}\left(\alpha_{i} u_{i}^{2}+\beta_{i} v_{i}^{2}+\gamma_{i} w_{i}^{2}\right),
$$

where the index $i$ refers to the level concerned and $\alpha_{i}$, $\beta_{i}, \gamma_{i}$ are weight factors.

The minimization of $F$ is subject to the restrictions that the differential equation (3) is to be satisfied at all levels. In order to express these restrictions, (3) must be expressed in terms of finite differences. Two choices for this transformation are obvious; either one makes use of a finite-difference approximation for the derivative, or one expresses (3) in integrated form and uses a quadrature formula for the integral.

To keep the arithmetic simple, the latter choice is made with the result that

$$
\begin{aligned}
U_{i+1}-U_{i}=\left(U_{i}+W_{i}-V_{i}\right) p_{i} & \\
& +\left(U_{i+1}+W_{i+1}-V_{i+1}\right) q_{i},
\end{aligned}
$$

where $p_{i}=\Delta z / 2 H_{p}(i)$ and $q_{i}=\Delta z / 2 H_{p}(i+1)$ are quadrature factors for the trapezoid rule combined with the pressure scale height at the level concerned. In terms of the given data values and the adjustments, the restrictions become

$u_{i+1}-u_{i}-\left(u_{i}+w_{i}-v_{i}\right) p_{i}$

$$
-\left(u_{i+1}+w_{i+1}-v_{i+1}\right) q_{\imath}+E_{i}^{0}
$$

where

$$
\begin{aligned}
E_{i}{ }^{0}=U_{i+1^{0}}-U_{i}^{0}-\left(U_{i}^{0}+W_{i}^{0}-V_{i}^{0}\right) p_{i} & \\
& -\left(U_{i+1}{ }^{0}+W_{i+1}^{0}-V_{i+1}{ }^{0}\right) q_{i}
\end{aligned}
$$

is the amount by which the observed values fail to satisfy (3a). The Lagrange multipliers, $\lambda_{i}$, are introduced so that the function to be minimized becomes

$$
\begin{aligned}
& F=\frac{1}{2} \sum_{i=1}^{n}\left(\alpha_{i} u_{i}{ }^{2}+\beta_{i} v_{i}{ }^{2}+\gamma_{i} w_{i}{ }^{2}\right) \\
&+\sum_{i=1}^{n-1} \lambda_{i}\left\{u_{i+1}-u_{i}-\left(u_{i}+w_{i}-v_{i}\right) p_{i}\right. \\
&\left.\quad-\left(u_{i+1}+w_{i+1}-v_{i+1}\right) q_{i}+E_{i}{ }^{0}\right\} .
\end{aligned}
$$

Differentiating (5) with respect to $u_{j}, v_{j}, w_{j}$ and equating the derivatives to zero leads to three systems of equations

$$
\left.\begin{array}{rl}
\alpha_{j} u_{j} & =\lambda_{j}\left(1+p_{j}\right)-\lambda_{j-1}\left(1-q_{j-1}\right) \\
\beta_{j} v_{j} & =-\lambda_{j-1} q_{j-1}-\lambda_{j} p_{j} \\
\gamma_{j} w_{j} & =\lambda_{j-1} q_{j-1}+\lambda_{j} p_{j}
\end{array}\right\} .
$$

The values of $u_{j}, v_{j}, w_{j}$ from (6) are substituted into the restrictions (4) to obtain a system of equations for the Lagrange multipliers:

$$
\begin{gathered}
-\lambda_{i-1}\left\{\left(1-q_{i-1}\right)\left(1+p_{i}\right) / \alpha_{i}-\left(1 / \beta_{i}+1 / \gamma_{i}\right) p_{i} q_{i-1}\right\} \\
+\lambda_{i}\left\{\left(1-q_{i}\right)^{2} / \alpha_{i+1}+\left(1+p_{i}\right)^{2} / \alpha_{i}+\left(1 / \beta_{i}+1 / \gamma_{i}\right) p_{i}{ }^{2}\right. \\
\left.+\left(1 / \beta_{i+1}+1 / \gamma_{i+1}\right) q_{i}{ }^{2}\right\}-\lambda_{i+1}\left\{\left(1-q_{i}\right)\left(1+p_{i+1}\right) / \alpha_{i+1}\right. \\
\left.-\left(1 / \beta_{i+1}+1 / \gamma_{i+1}\right) p_{i+1} q_{i}\right\}=E_{i}{ }^{0}
\end{gathered}
$$

One now solves the system of equations (7) for the Lagrange multipliers, $\lambda_{i}$, and substitutes these values into (6) to obtain the adjustments of the data, $u_{i}, v_{i}$, $w_{i}$, which minimize the function (5). The reader will recognize that (6) and (7) must be suitably modified for values at the beginning and end of the sequence of data levels (to avoid values $\lambda_{0}$ and $\lambda_{n}$ which are not defined).

\section{Application}

Smith and Johnson (1969, pp. 14.12-14.13) tabulate values of the coefficients of variation, $\sigma_{p} / \bar{p}, \sigma_{\rho} / \bar{\rho}, \sigma_{T} / \bar{T}$, and the correlation coefficients $r_{p T}, r_{T_{\rho}}, r_{\rho p}$ from the surface to $90 \mathrm{~km}$ over Cape Kennedy, Fla. The adjustment procedure of the preceding section was applied to these data. That some adjustment is required is apparent by inspection. From (2b) it is obvious that the correlation coefficient relating pressure and temperature, $r_{p T}$, should have the same sign as the slope of $\sigma_{p} / \bar{p}$. This is only partially the case.

The original and adjusted values of the coefficient of variation are shown in Fig. 1. To approximate the likely accuracy of the data, weight factors were assigned as follows: 10 . from $0-20 \mathrm{~km}, 1$. from $25-90 \mathrm{~km}$, and a linear change from 10 . to 1 . on the range $20-25 \mathrm{~km}$. The weight factors were of little importance since the results with a weight of 1 . for the entire range are scarcely distinguishable from those with the variable weights.

The adjustments of the coefficients of variation appear to be reasonable and compatible within the accuracy of the initial data. The effect of these adjustments on the correlation coefficients is much more dramatic. The original correlation coefficients and those computed from the adjusted coefficients of variation are shown in Fig. 2. The most important changes are in the coefficient of variation for pressure, $\sigma_{p} / \bar{p}$, and the correlation coefficient relating pressure and temperature, $r_{p T}$. The minor local maximum of $\sigma_{p} / \bar{p}$ at $3 \mathrm{~km}$ is eliminated and the curve is smoothed to give a minimum near $2 \mathrm{~km}$. This results in agreement between the sign of $r_{p T}$ and the slope of $\sigma_{p} / \bar{p}$ with quite insignificant changes in the values of $r_{p T}$. The slope of $\sigma_{p} / \bar{p}$ is positive for adjusted values from $20-68 \mathrm{~km}$ while that of the original data has a pronounced negative slope on the range $33-37 \mathrm{~km}$ and positive from $37-68 \mathrm{~km}$. The adjusted values of $r_{p T}$ are positive on this range (as they should be). The unadjusted values of $r_{p T}$ become negative at $35 \mathrm{~km}$ and remain negative to $71 \mathrm{~km}$ rather than becoming positive again where the slope of $\sigma_{p} / \bar{p}$ is positive. The alternating negative and positive slope 


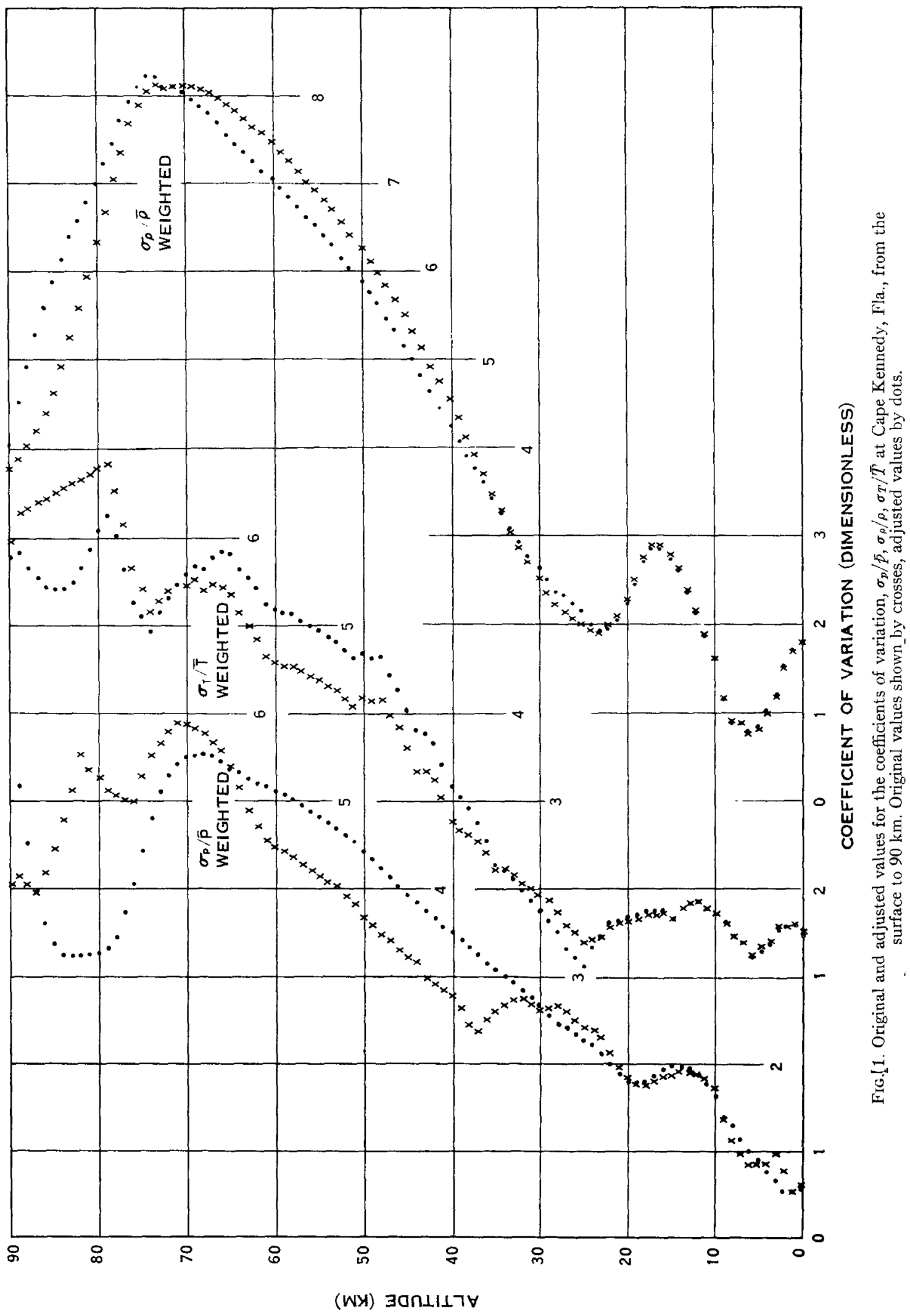




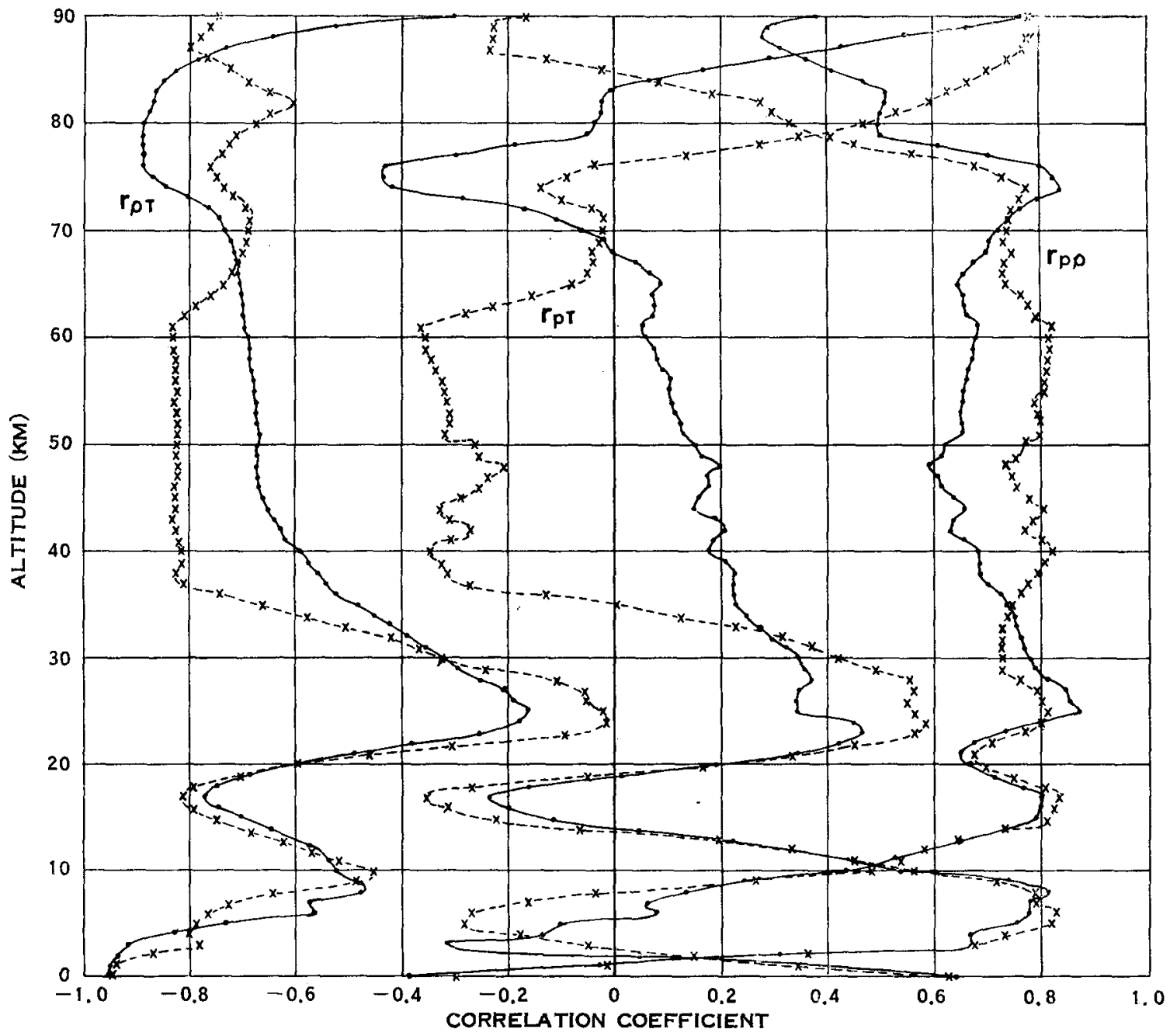

Fig. 2. Original and adjusted values for the correlation coefficients $r_{p}, r_{p}, r_{p p}$ at Cape Kennedy, Fla., from the surface to 90 $\mathrm{km}$. Original values shown by connected crosses, adjusted values by the connected dots.

of $\sigma_{p} / \bar{p}$ in the range from $70-90 \mathrm{~km}$ (before adjustment) should be accompanied by alternating signs of $r_{p r}$ (unadjusted) whereas these values become positive and remain so. The sign of the slope for $\sigma_{p} / \bar{p}$ (adjusted) and the sign of $r_{p T}$ (adjusted) agree. The above has been expressed only in terms of the signs. Actually the adjusted values satisfy (2b) in detail.

Although some significant adjustments of the coefficients of variation are made at levels below $25 \mathrm{~km}$, these are all small. The larger adjustments occur at the higher levels where the original data are less reliable. This is not due to the smaller weights given to the data above $25 \mathrm{~km}$ since almost exactly the same adjustments were made when uniform weights were assigned throughout the entire data range.

\section{Depth of pressure systems}

The hydrostatic equation in the form of Eq. (2) may be used to derive an expression for the "depth" of pressure perturbation systems. This depth parameter $D$ appears in the representation of the two-point correlation coefficient for pressure as

$$
r_{p p^{\prime}}=1-\left(z^{\prime}-z\right)^{2} / 2 D^{2}+\cdots,
$$

where $z$ and $z^{\prime}$ are the altitudes at which the pressure perturbations $p$ and $p^{\prime}$ are observed. The two-point pressure correlation coefficient is constrained to have this behavior for small values of $\left(z^{\prime}-z\right)$ since the hydrostatic equation implies that pressure is a differentiable function in the vertical. The depth of pressure perturbation systems is related to the coefficients of variation for pressure and temperature by

$$
\left\{\partial\left(\sigma_{p} / \bar{p}\right) / \partial z\right\}^{2}+\left(\sigma_{p} / \bar{p}\right)^{2} / D^{2}=\left(\sigma_{T} / \bar{T}\right)^{2} / H_{p}^{2}
$$

The method for obtaining this relation is given in Buell (1954).

Using (2b) and (8), the depth of pressure systems may be written as 


$$
D=H_{p}\left(\sigma_{p} / \bar{p}\right) /\left[\left(\sigma_{T} / \bar{T}\right)\left(1-r_{p} T^{2}\right)^{\frac{1}{2}}\right] .
$$

Consequently, the depth of pressure systems is proportional to the ratio of the coefficient of variation of pressure to that of temperature, and it involves the correlation coefficient relating pressure and temperature at a point in such a way that the depth is minimum when this correlation coefficient is zero and becomes large without bound when it approaches +1 or -1 .

The depth of pressure systems is shown in Fig. 3 based on adjusted values of the coefficients of variation and the corresponding correlation coefficient for pressure and temperature. The variation of $D$ with height agrees with the well-known facts in the range from 0 to $30 \mathrm{~km}$ : shallow pressure systems near the ground, deep in the upper troposphere, shallow in the lower stratosphere, and becoming quite deep near $25 \mathrm{~km}$. Above this level the depth $D$ decreases steadily to $75 \mathrm{~km}$. The decrease in depth to very shallow pressure perturbations near $80-85 \mathrm{~km}$ and abrupt increase from $85-90 \mathrm{~km}$ reflects the structure of $\left(\sigma_{p} / \bar{p}\right) /\left(\sigma_{T} / \bar{T}\right)$ at those levels. This same behavior of $D$ would be present if the original data had been used.

\section{Remarks}

The method for adjusting the values of the coefficients of variation described in the preceding section is in complete since there are further restrictions that must be applied. It was pointed out in Buell (1970) that the coefficients of variation are subject to the "triangle inequalities," $\sigma_{p} / \bar{p} \leqslant \sigma_{\rho} / \bar{\rho}+\sigma_{T} / \bar{T}, \quad \sigma_{\rho} / \bar{\rho} \leqslant \sigma_{T} / \bar{T}+\sigma_{p} / \bar{p}$, $\sigma_{w} / \bar{T} \leqslant \sigma_{p} / \bar{p}+\sigma_{p} / \bar{\rho}$. The violation of these restrictions would result in a correlation coefficient, $r_{p T}, r_{T \rho}, r_{\rho p}$ being greater than 1 in absolute value. We are probably only lucky that such a correlation coefficient was not encountered in the example discussed here. Also, the adjusted values of the coefficients of variation must all be positive. The application of these additional restrictions leads to a vastly more complex minimization problem.

In their discussion of the relation

$$
\partial \sigma_{p} / \partial z=-g \sigma_{\rho} r_{p p}
$$

where $r_{p \rho}$ is the correlation coefficient between density and pressure at a point, Minzner and Morgenstern (1971) use the second-order central-difference formula to approximate the derivative of the standard deviation of pressure. They found that this finite-difference formulation was not as well satisfied by the data as the corresponding integrated formulation in finite-difference terms. This is at least partially due to the fact that the higher order finite-difference formulas when applied to "noisy" data tend to amplify the "noise." A smoothed estimate of the derivative would have given results more nearly in agreement with the integrated formulation.

The expression of the statistical relations (2b) and (3) in terms of the coefficients of variation permits the

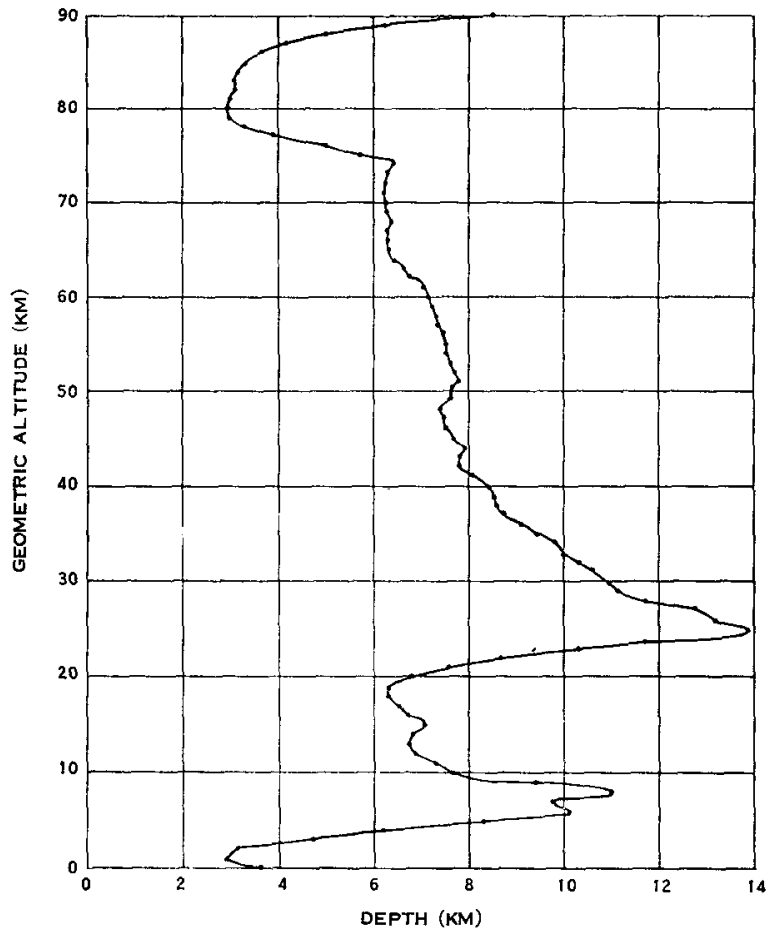

FIG. 3. Values of the depth of pressure perturbation systems at Cape Kennedy, Fla., from the surface to $90 \mathrm{~km}$.

use of quantities that have a range from $1 \%$ to $10 \%$ rather than a range of many orders of magnitude that are encountered in (10). This greatly simplifies the manipulations involved and makes the elaborate quadrature formulas of Minzner and Morgenstern unnecessary. The use of the coefficients of variation in relations (2b) and (3), where geometric altitude is the independent variable, has some of the advantages present in the description of the statistical state of the atmosphere in terms of geopotential and temperature on isobaric surfaces.

The quadrature formulation (3a) for (3) is chosen so as to use data from two adjacent levels throughout the data range in order to preserve as much of the detail shown in the original data as possible. The adjusted values shown in Fig. 1 show that the values are smoothed to some extent.

Eq. (3) may be handled as a Bernoulli equation in the dependent variable $U$ with $(W-V)$ serving as a "forcing function." This provides a third approach to the method of making adjustments to the original data. Other methods that might be used include a modified method of Kalman filtering and the method of sensitivity coefficients (Radix, 1970). Adjustment using (2b) directly is also possible.

Acknoweledgments. We wish to thank Mr. O. E. Smith (NASA-Huntsville) and Col. Thomas D. Potter (ETAC) and their staffs who were so kind as to review 
this paper and make several helpful suggestions. The suggestions of the referees have also been helpful.

\section{REFERENCES}

Buell, C. E., 1954: Some relations among atmospheric statistics. J. Meteor., 2, 238-244.

-, 1970 : Statistical relations in a perfect gas. J. Appl. Meteor., 9, 729-731.
Minzner, R. A., and P. Morgenstern. 1971: Analysis of several relations among atmospheric statistics. NASA Tech. Rept. TR R-365, $39 \mathrm{pp}$.

Radix, J. C., 1970: Intraduction au Filtrage Numérique. Paris, Editions Eyrolles, $240 \mathrm{pp}$.

Smith, Orvel E., and Dale L. Johnson, 1969: Inflight thermodynamic properties. Section X1V, Terrestrial environment (climatic) criteria guidelines for use in space vehicle devclopment, 1969 Revision. NASA Tech. Memo., Rept. TM X64589, George C. Marshall Space Lilight Center, Ala. 